Supplement of Clim. Past, 16, 1777-1805, 2020

https://doi.org/10.5194/cp-16-1777-2020-supplement

(c) Author(s) 2020. This work is distributed under

the Creative Commons Attribution 4.0 License.

(c) (1)

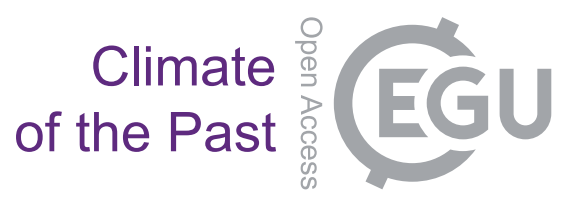

Supplement of

\title{
Comparison of past and future simulations of ENSO in CMIP5/PMIP3 and CMIP6/PMIP4 models
}

\section{Josephine R. Brown et al.}

Correspondence to: Josephine R. Brown (josephine.brown@unimelb.edu.au)

The copyright of individual parts of the supplement might differ from the CC BY 4.0 License. 
Table S1: Details of CMIP6/PMIP4 models included in this study: Model name, institution, reference, atmosphere resolution, ocean resolution.

\begin{tabular}{|c|c|c|c|c|c|c|c|c|c|c|}
\hline \multirow[b]{2}{*}{ Model name } & \multirow[b]{2}{*}{ Institution } & \multirow[b]{2}{*}{ References } & \multicolumn{4}{|c|}{ Atmosphere resolution } & \multicolumn{4}{|c|}{ Ocean resolution } \\
\hline & & & Lon & Lat & Level & $\begin{array}{l}\text { Top } \\
\text { level }\end{array}$ & Lon & Lat & Level & $\begin{array}{l}\text { Top } \\
\text { grid cell }\end{array}$ \\
\hline $\begin{array}{l}\text { AWI-ESM-1-1- } \\
\text { LR }\end{array}$ & $\begin{array}{l}\text { Alfred Wegener } \\
\text { Institute (AWI), } \\
\text { Germany }\end{array}$ & $\begin{array}{l}\text { Sidorenko et } \\
\text { al. (2015) \& } \\
\text { Rackow et al. } \\
\text { (2018); Danek } \\
\text { et al. } \\
\text { (2020a,b,c); } \\
\text { Shi et al. } \\
\text { (2020a,b,c,d) }\end{array}$ & 192 & 96 & 47 & $80 \mathrm{~km}$ & $\begin{array}{l}1268 \\
\text { node }\end{array}$ & wet & 46 & $0-5 \mathrm{~m}$ \\
\hline CNRM-CM6-1 & $\begin{array}{l}\text { CNRM- } \\
\text { CERFACS, } \\
\text { France }\end{array}$ & $\begin{array}{l}\text { Voldoire et al. } \\
\text { (2019) }\end{array}$ & 256 & 128 & 91 & $78.4 \mathrm{~km}$ & 362 & 294 & 75 & $0-1 \mathrm{~m}$ \\
\hline CESM2 & $\begin{array}{l}\text { National Center } \\
\text { for Atmospheric } \\
\text { Research } \\
\text { (NCAR), USA }\end{array}$ & $\begin{array}{l}\text { Danabasoglu et } \\
\text { al. }(2020)\end{array}$ & 288 & 192 & 32 & $2.25 \mathrm{mb}$ & 320 & 384 & 60 & $0-10 \mathrm{~m}$ \\
\hline EC-EARTH3-LR & $\begin{array}{l}\text { EC-Earth } \\
\text { Consortium }\end{array}$ & $\begin{array}{l}\text { Doescher et al. } \\
\text { (in preparation) }\end{array}$ & 320 & 160 & 62 & $5 \mathrm{hPa}$ & 362 & 292 & 75 & $0-1 \mathrm{~m}$ \\
\hline FGOALS-f3-L & $\begin{array}{l}\text { Chinese } \\
\text { Academy of } \\
\text { Sciences } \\
\text { (CAS), China } \\
\end{array}$ & $\begin{array}{l}\text { He et al. } \\
\text { (2019) }\end{array}$ & 360 & 180 & 32 & $2.16 \mathrm{hPa}$ & 360 & 218 & 30 & $0-10 \mathrm{~m}$ \\
\hline FGOALS-g3 & $\begin{array}{l}\text { Chinese } \\
\text { Academy of } \\
\text { Sciences } \\
\text { (CAS), China }\end{array}$ & Li et al. (2020) & 180 & 80 & 26 & $2.19 \mathrm{hPa}$ & 360 & 218 & 30 & $0-10 \mathrm{~m}$ \\
\hline GISS-E2-1-G & $\begin{array}{l}\text { NASA Goddard } \\
\text { Institute for } \\
\text { Space Studies } \\
\text { (GISS), USA }\end{array}$ & $\begin{array}{l}\text { Kelley et al. } \\
\text { (submitted) }\end{array}$ & 144 & 90 & 40 & $0.1 \mathrm{hPa}$ & 360 & 180 & 40 & $0-10 \mathrm{~m}$ \\
\hline $\begin{array}{l}\text { HadGEM3- } \\
\text { GC31-LL }\end{array}$ & $\begin{array}{l}\text { Met. Office } \\
\text { Hadley Centre, } \\
\text { UK }\end{array}$ & $\begin{array}{l}\text { Williams et al. } \\
(2018)\end{array}$ & 192 & 144 & 85 & $85 \mathrm{~km}$ & 360 & 330 & 75 & $0-1 \mathrm{~m}$ \\
\hline INM-CM4-8 & $\begin{array}{l}\text { Institute of } \\
\text { Numerical } \\
\text { Mathematics of } \\
\text { the Russian } \\
\text { Academy of } \\
\text { Sciences, } \\
\text { Russia }\end{array}$ & $\begin{array}{l}\text { Volodin et al. } \\
\text { (2018) }\end{array}$ & 180 & 120 & 21 & $\begin{array}{l}\text { sigma }= \\
0.01\end{array}$ & 360 & 318 & 40 & $\begin{array}{l}0.001426 \\
\text { sigma }\end{array}$ \\
\hline IPSL-CM6A-LR & $\begin{array}{l}\text { Institut Pierre } \\
\text { Simon Laplace } \\
\text { (IPSL), France }\end{array}$ & $\begin{array}{l}\text { Boucher et al. } \\
(2020)\end{array}$ & 144 & 143 & 79 & $40 \mathrm{~km}$ & 362 & 332 & 75 & $0-2 m$ \\
\hline MPI-ESM1-2-LR & $\begin{array}{l}\text { Max Planck } \\
\text { Institute for } \\
\text { Meteorology } \\
\text { (MPI), } \\
\text { Germany }\end{array}$ & $\begin{array}{l}\text { Mauritsen et al. } \\
\text { (2019) }\end{array}$ & 192 & 96 & 47 & $0.01 \mathrm{hPa}$ & 256 & 220 & 40 & $0-12 \mathrm{~m}$ \\
\hline
\end{tabular}




\begin{tabular}{|c|c|c|c|c|c|c|c|c|c|c|}
\hline MIROC-ES2L & $\begin{array}{l}\text { Japan Agency } \\
\text { for Marine- } \\
\text { Earth Science } \\
\text { and } \\
\text { Technology, } \\
\text { Atmosphere and } \\
\text { Ocean Research } \\
\text { Institute } \\
\text { (University of } \\
\text { Tokyo), and } \\
\text { National } \\
\text { Institute for } \\
\text { Environmental } \\
\text { Studies, Japan }\end{array}$ & $\begin{array}{l}\text { Hajima et al. } \\
\text { (2020); } \\
\text { Ohgaito et al. } \\
\text { (in review) }\end{array}$ & 128 & 64 & 40 & $3 \mathrm{hPa}$ & 360 & 256 & 63 & $0-2 m$ \\
\hline MRI-ESM2-0 & $\begin{array}{l}\text { Meteorological } \\
\text { Research } \\
\text { Institute (MRI), } \\
\text { Japan }\end{array}$ & $\begin{array}{l}\text { Yukimoto et al. } \\
(2019)\end{array}$ & 320 & 160 & 80 & $0.01 \mathrm{hPa}$ & 360 & 364 & 61 & $0-2 m$ \\
\hline NESM3 & $\begin{array}{l}\text { Nanjing } \\
\text { University of } \\
\text { Information } \\
\text { Science and } \\
\text { Technology, } \\
\text { China }\end{array}$ & $\begin{array}{l}\text { Cao et al. } \\
(2018)\end{array}$ & 192 & 96 & 47 & $1 \mathrm{~Pa}$ & 384 & 362 & 46 & $0-6 \mathrm{~m}$ \\
\hline NorESM1-F & $\begin{array}{l}\text { Bjerknes Centre } \\
\text { for Climate } \\
\text { Research, } \\
\text { Norway }\end{array}$ & $\begin{array}{l}\text { Guo et al. } \\
\text { (2019) }\end{array}$ & 144 & 96 & 32 & $3 \mathrm{mb}$ & 360 & 384 & 70 & $0-2.5 \mathrm{~m}$ \\
\hline NorESM2-LM & $\begin{array}{l}\text { Bjerknes Centre } \\
\text { for Climate } \\
\text { Research, } \\
\text { Norway }\end{array}$ & $\begin{array}{l}\text { Seland et al. } \\
(2020)\end{array}$ & 144 & 96 & 32 & $3 \mathrm{mb}$ & 360 & 384 & 70 & $0-2.5 \mathrm{~m}$ \\
\hline UofT-CCSM-4 & $\begin{array}{l}\text { University of } \\
\text { Toronto, } \\
\text { Canada }\end{array}$ & $\begin{array}{l}\text { Chandan and } \\
\text { Peltier (2017) }\end{array}$ & 288 & 192 & 26 & $2 \mathrm{hPa}$ & 384 & 320 & 60 & $0-10 \mathrm{~m}$ \\
\hline
\end{tabular}



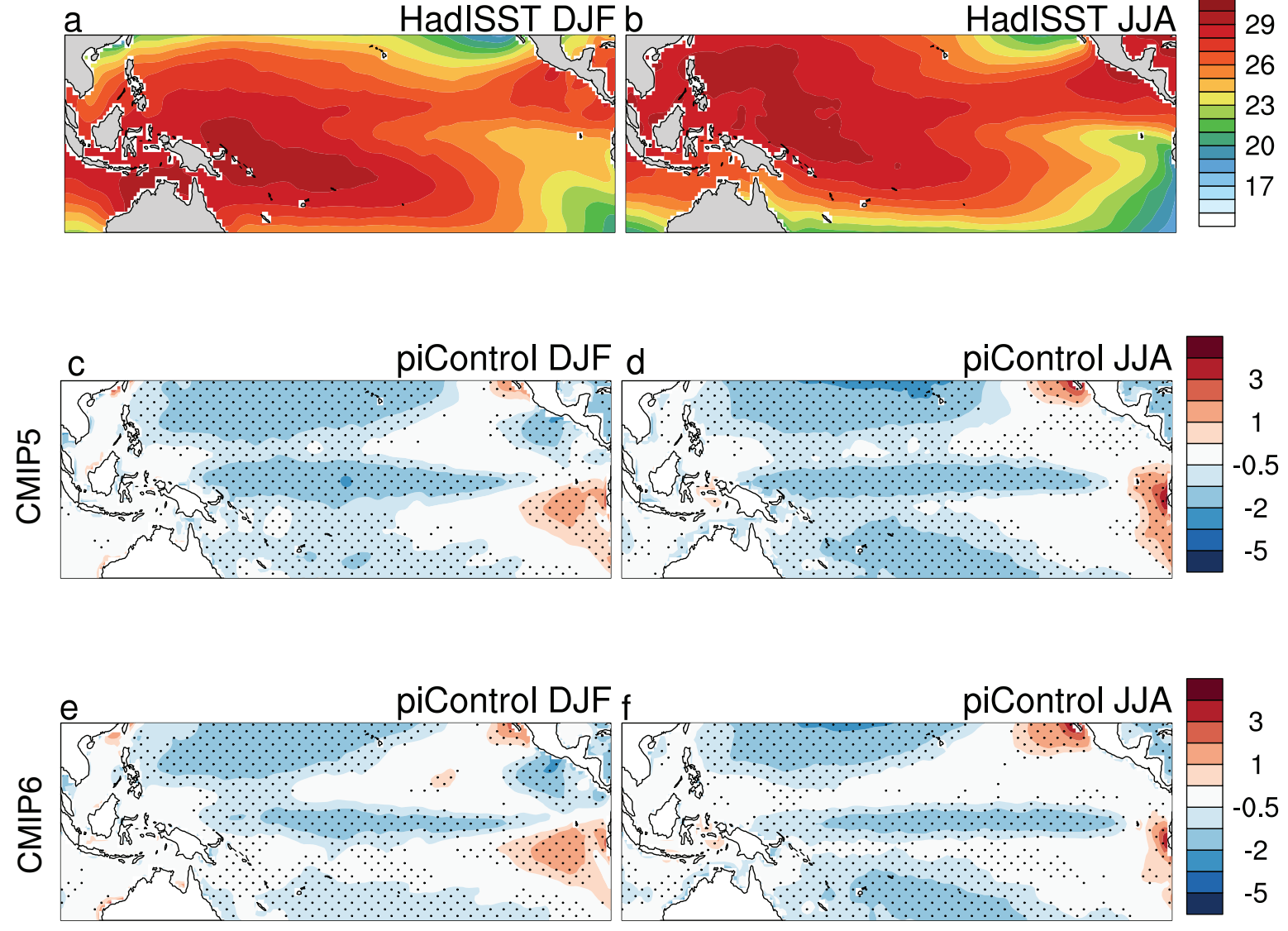

Figure S1: Sea surface temperature evaluation: Observed HadISST for (a) DJF and (b) JJA, CMIP5 piControl multi-model mean anomaly relative to HadISST for (c) DJF and (d) JJA and CMIP6 piControl multi-model mean anomaly relative to HadISST for (e) DJF and (f) JJA. Stippling indicates $2 / 3$ of models agree on sign of anomaly. Units are ${ }^{\circ} \mathrm{C}$. 

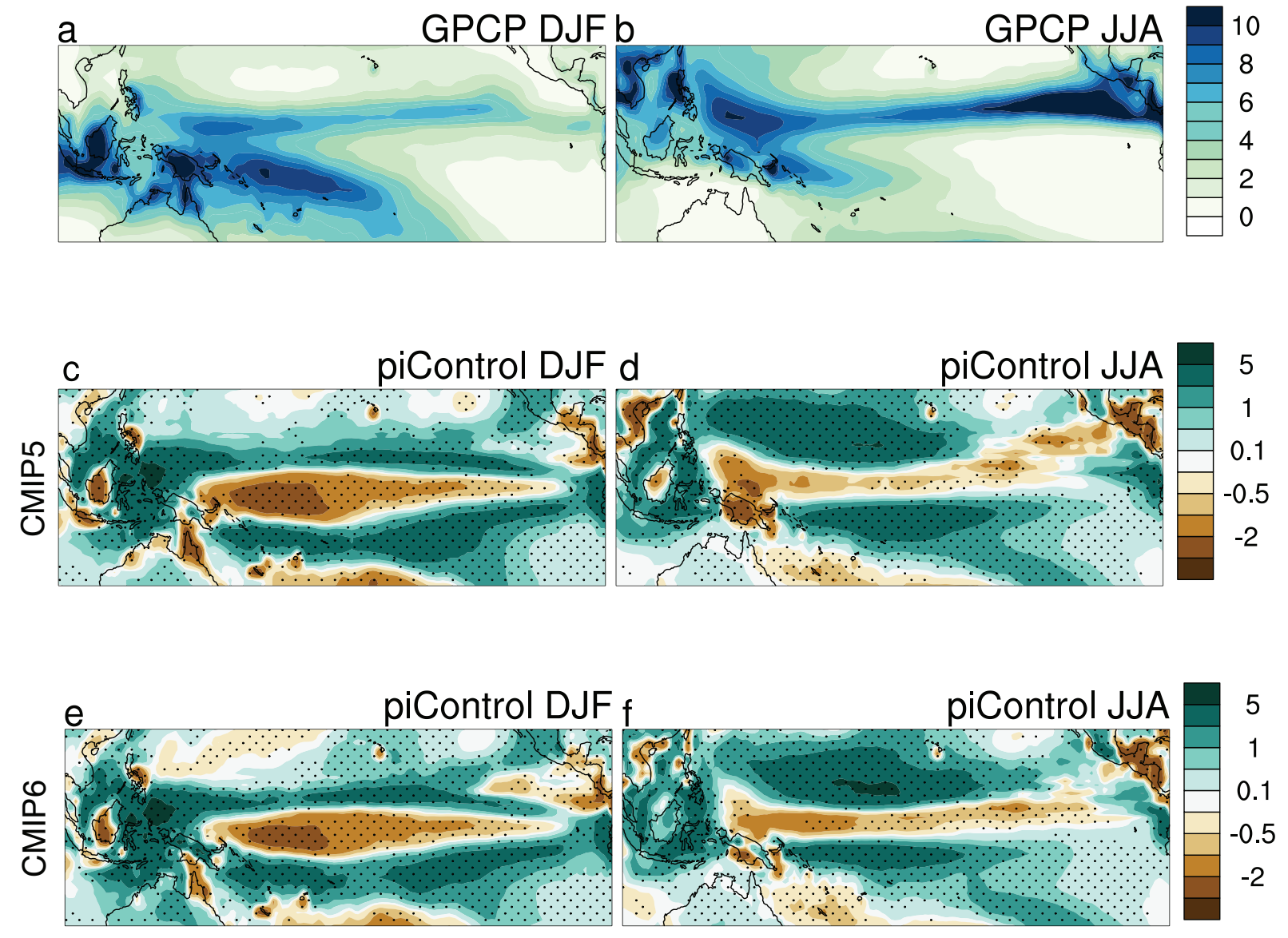

Figure S2: Precipitation evaluation: Observed GPCP for (a) DJF and (b) JJA, CMIP5 piControl multi-model mean anomaly relative to GPCP for (c) DJF and (d) JJA and CMIP6 piControl multi-model mean anomaly relative to GPCP for (e) DJF and (f) JJA. Stippling indicates $2 / 3$ of models agree on sign of anomaly. Units are $\mathrm{mm} /$ day. 
a

HadISST

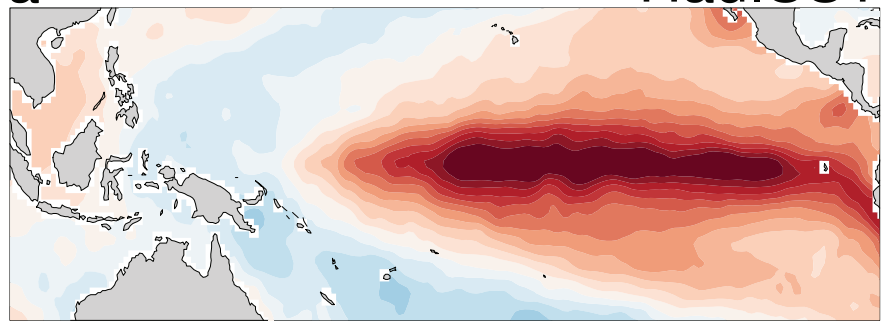

b

piControl
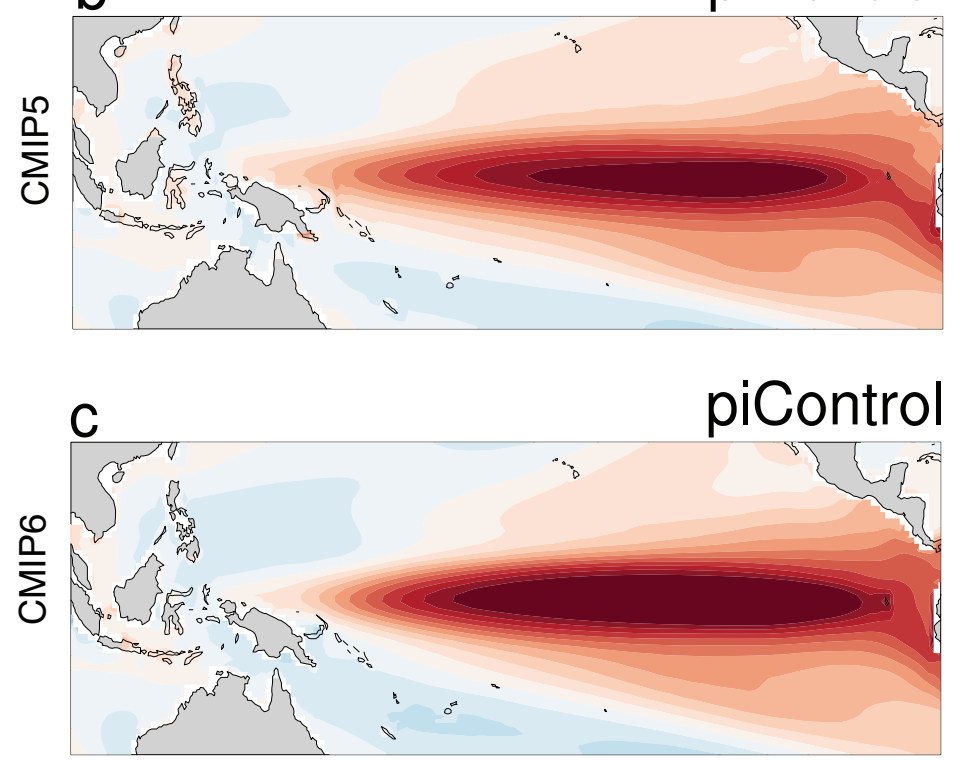

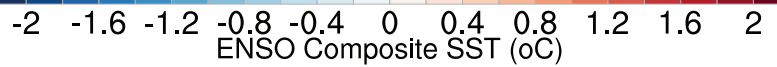

Figure S3: Evaluation of ENSO SST anomaly pattern: Composite El Niño minus La Niña sea surface temperature anomaly from (a) HadISST observations, (b) CMIP5 model ensemble average from piControl simulations and (c) CMIP6 model ensemble average from piControl simulations. Units are ${ }^{\circ} \mathrm{C}$. 


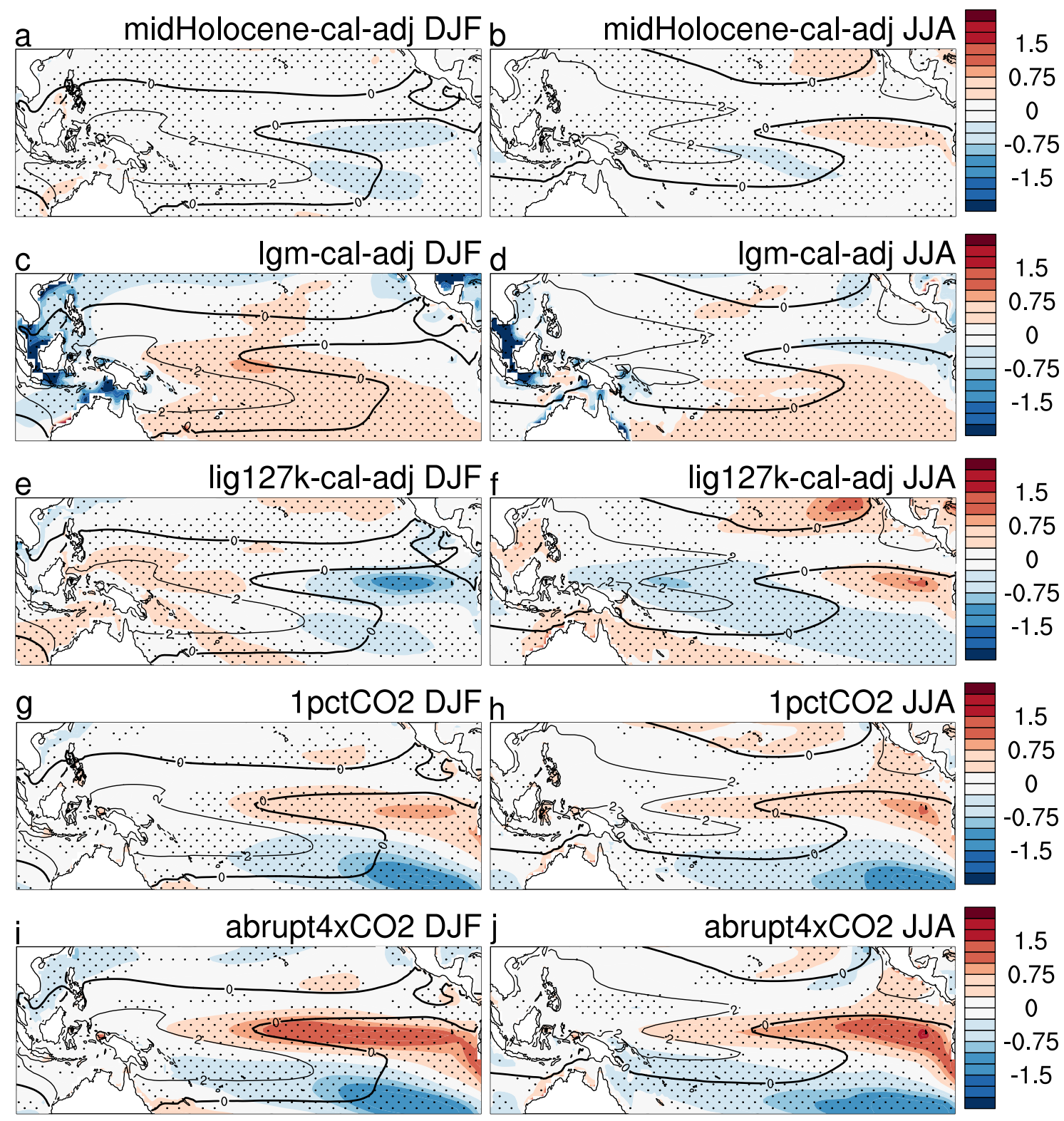

Figure S4: Ensemble mean seasonal changes in sea surface temperature in experiments minus pre-industrial control simulations as for Figure 6 but with tropical mean SST change (over $25^{\circ} \mathrm{N}-25^{\circ} \mathrm{S}, 100^{\circ} \mathrm{E}-80^{\circ} \mathrm{W}$ ) subtracted. The ensemble mean temperature pattern in the pre-industrial control simulations is shown as black contours. Units are ${ }^{\circ} \mathrm{C}$. Stippling indicates that more than $2 / 3$ of the ensemble members agree on the sign of the change. 

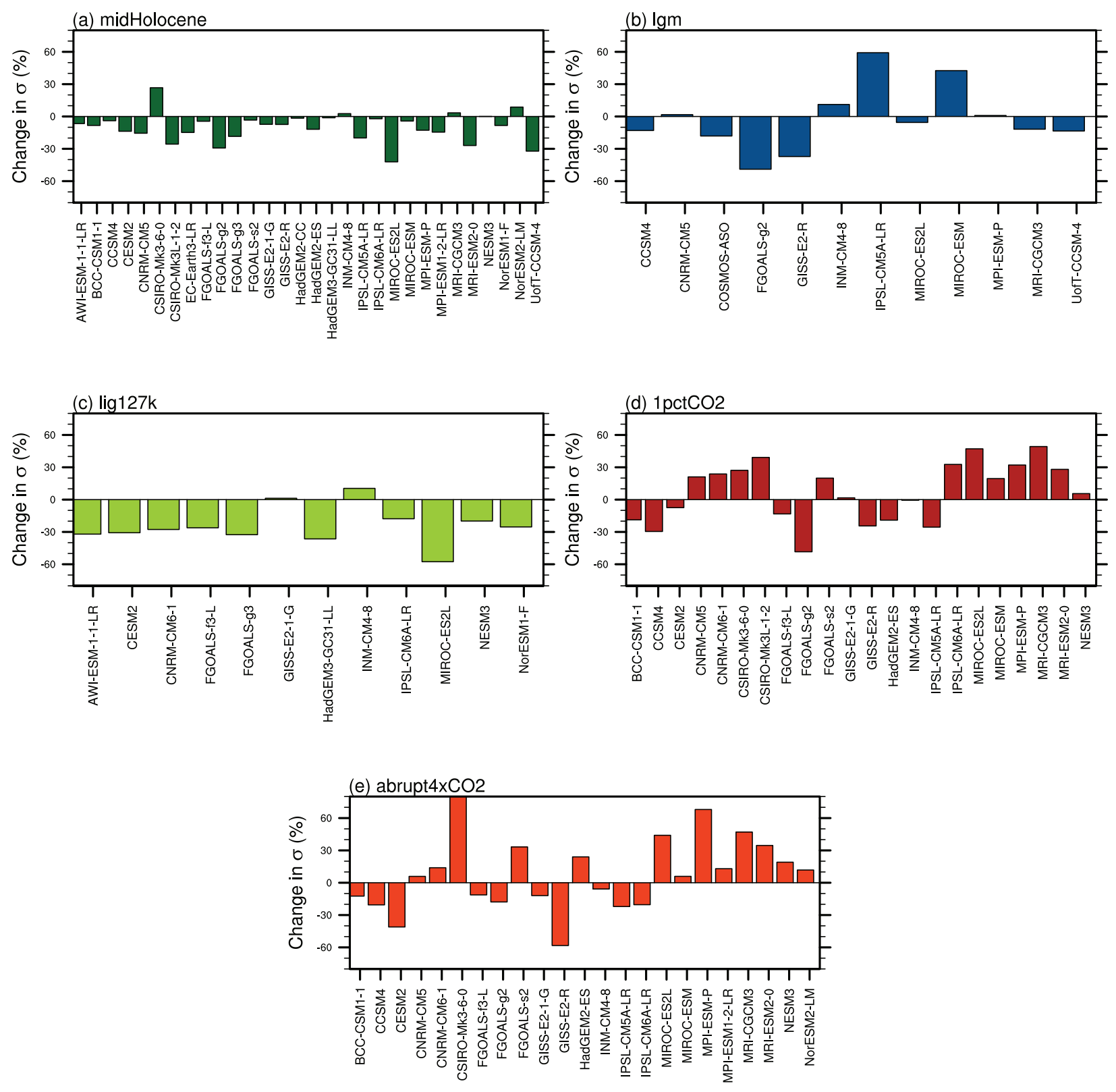

Figure S5: Change in amplitude of ENSO measured from standard deviation of 2-8 year bandpass filtered NINO3.4 index relative to piControl amplitude (\%) in (a) midHolocene, (b) lgm, (c) lig127k, (d) $1 p c t C O 2$ and (e) abrupt4xCO2. Model names are given below plots. As in Figure 9 but using bandpass filtered NINO3.4 indices. 
(a) CMIP5 midHolocene

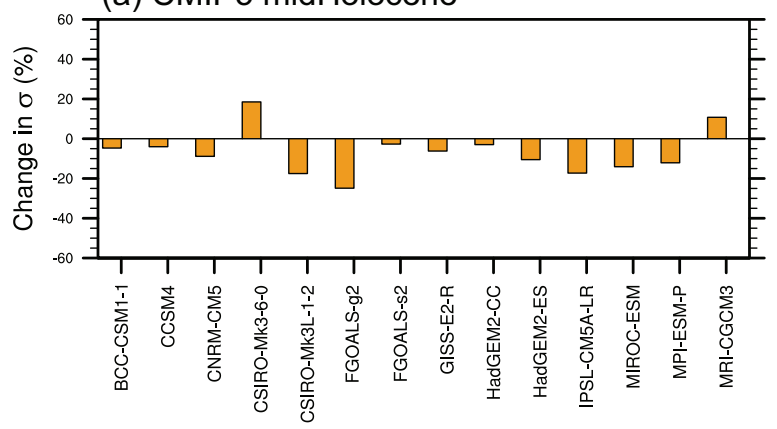

(c) CMIP5 LGM

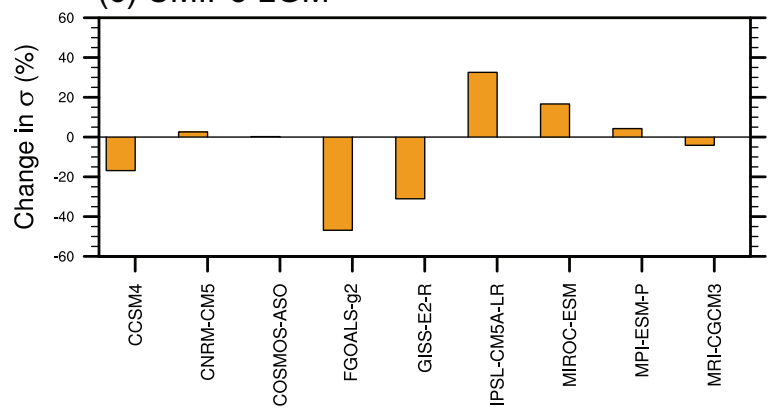

(e) CMIP5 abrupt4xCO2

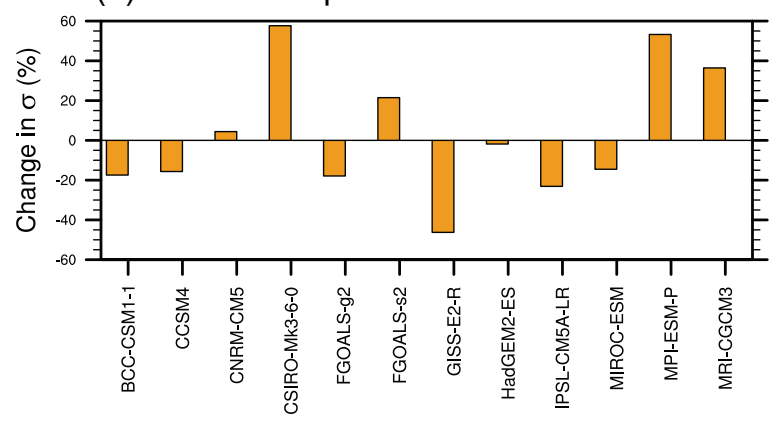

(b) CMIP6 midHolocene

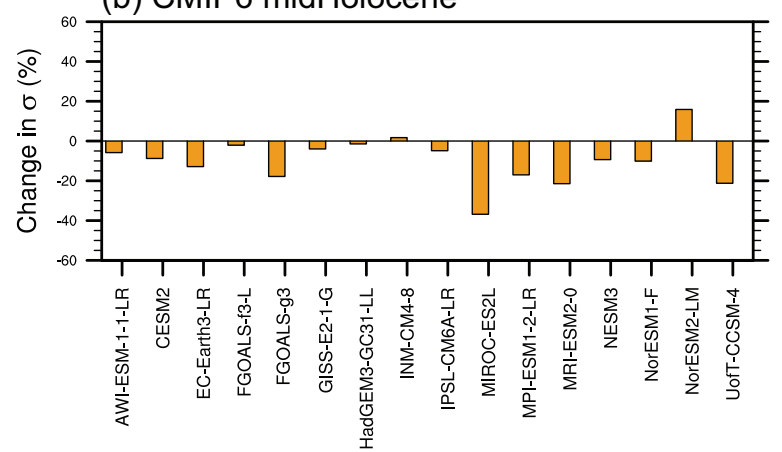

(d) CMIP6 LGM

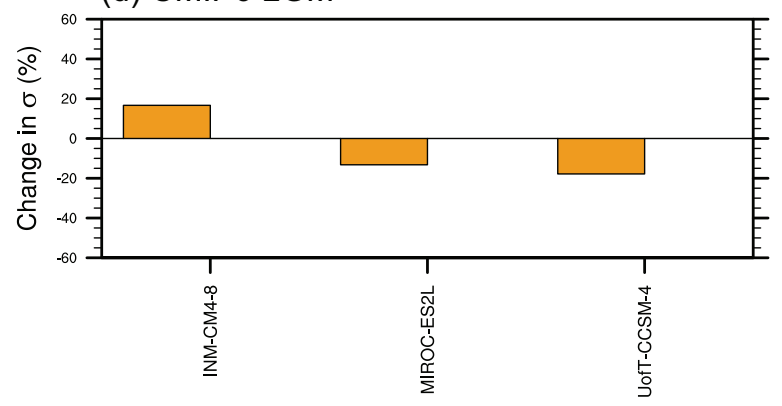

(f) CMIP6 abrupt4xCO2

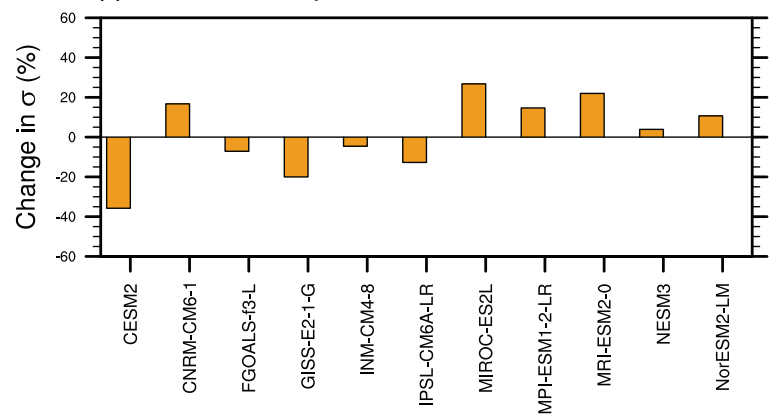

Figure S6: Change in standard deviation of NINO3.4 SST (\%) relative to piControl for (a) CMIP5 midHolocene and (b) CMIP6 midHolocene, (c) CMIP5 lgm and (d) CMIP6 lgm and (e) CMIP5 abrupt4xCO2 and (f) CMIP6 abrupt4xCO2 experiments. 


\section{References:}

Boucher, O., Servonnat, J., Albright, A. L., Aumont, O., Balkanski, Y., Bastrikov, V., Bekki, S., Bonnet, R., Bony, S., Bopp, L., Braconnot, P.,Brockmann, P., Cadule, P., Caubel, A., Cheruy, F., Cozic, A., Cugnet, D., D’Andrea, F., Davini, P., de Lavergne, C., Denvil, S., Deshayes,J., Devilliers, M., Ducharne, A., Dufresne, J.-L., Dupont, E., Éthé, C., Fairhead, L., Falletti, L., Foujols, M.-A., Gardoll, S., Gastineau, G.,Ghattas, J., Grandpeix, J.-Y., Guenet, B., Guez, L., Guilyardi, E., Guimberteau, M., Hauglustaine, D., Hourdin, F., Idelkadi, A., Joussaume,S., Kageyama, M., Khodri, M., Krinner, G., Lebas, N., Levavasseur, G., Lévy, C., Li, L., Lott, F., Lurton, T., Luyssaert, S., Madec, G.,Madeleine, J.-B., Maignan, F., Marchand, M., Marti, O., Mellul, L., Meurdesoif, Y., Mignot, J., Musat, I., Ottlé, C., Peylin, P., Planton,Y., Polcher, J., Rio, C., Rousset, C., Sepulchre, P., Sima, A., Swingedouw, D., Thiéblemont, R., Traoré, A.-K., Vancoppenolle, M., Vial J., Vialard, J., Viovy, N., and Vuichard, N.: Presentation and evaluation of the IPSL-CM6A-LR climate model, J. Adv. Model. Earth Sy., 12, e2019MS002010. https://doi.org/10.1029/2019MS002010, 2020.

Cao, J., Wang, B., Yang, Y.-M., Ma, L., Li, J., Sun, B., Bao, Y., He, J., Zhou, X., and Wu, L.: The NUIST Earth System Model (NESM) version 3: description and preliminary evaluation, Geosci. Model Dev., 11, 2975-2993, https://doi.org/10.5194/gmd-112975-2018, 2018.

Chandan, D. and Peltier, W. R.: Regional and global climate for the mid-Pliocene using the University of Toronto version of CCSM4 and PlioMIP2 boundary conditions, Clim. Past, 13, 919-942, https://doi.org/10.5194/cp-13-919-2017, 2017.

Danabasoglu, G., Lamarque, J. F., Bachmeister, J., Bailey, D. A., DuVivier, A. K., Edwards, J., Emmons, L. K., Fasullo, J., Garcia, R.,Gettelman, A., Hannay, C., Holland, M. M., Large, W. G., Lawrence, D. M., Lenaerts, J. T. M., Lindsay, K., Lipscomb, W. H., Mills,M. J., Neale, R., Oleson, K. W., Otto-Bliesner, B., Phillips, A. S., Sacks, W., Tilmes, S., van Kampenhout, L., Vertenstein, M., Bertini,A., Dennis, J., Deser, C., Fischer, C., Fox-Kember, B., Kay, J. E., Kinnison, D., Kushner, P. J., Long, M. C., Mickelson, S., Moore, J. K.,Nienhouse, E., Polvani, L., Rasch, P. J., and Strand, W. G.: The Community Earth System Model Version 2 (CESM2), Journal of Advances in Modeling Earth Systems, 12, e2019MS001916, 2020

Danek, C., Shi, X., Stepanek, C. Yang, H., Barbi, D., Hegewald, J., Lohmann, G.: AWI-ESM1.1LR model output prepared for CMIP6 piControl. Earth System Grid Federation, https://doi.org/10.22033/ESGF/CMIP6.9335, 2020 a.

Danek, C., Shi, X., Stepanek, C. Yang, H., Barbi, D., Hegewald, J., Lohmann, G.: AWI-ESM1.1LR model output prepared for CMIP6 CMIP historical. Earth System Grid Federation, https://doi:10.22033/ESGF/CMIP6.9328, 2020 b.

Danek, C., Shi, X., Stepanek, C. Yang, H., Barbi, D., Hegewald, J., Lohmann, G.: AWI-ESM1.1LR model output prepared for CMIP6. Earth System Grid Federation, https://doi:10.22033/ESGF/CMIP6.9301, 2020c.

Doescher R and the EC-Earth Consortium.: The EC-Earth3 earth system model for the climate model intercomparison project 6, in preparation.

Guo, C., Bentsen, M., Bethke, I., Ilicak, M., Tjiputra, J., Toniazzo, T., Schwinger, J., and Otterå, O. H.: Description and evaluation of NorESM1-F: a fast version of the Norwegian Earth System Model (NorESM), Geosci. Model Dev., 12, 343-362, https://doi.org/10.5194/gmd-12-343-2019, 2019.

Hajima, T., Watanabe, M., Yamamoto, A., Tatebe, H., Noguchi, M. A., Abe, M., Ohgaito, R., Ito, A., Yamazaki, D., Okajima, H., Ito, A., Takata, K., Ogochi, K., Watanabe, S., and Kawamiya, M.: Development of the MIROC-ES2L Earth system model and the evaluation of biogeochemical processes and feedbacks, Geosci. Model Dev., 13, 2197-2244, https://doi.org/10.5194/gmd-132197-2020, 2020.

He, B., Bao, Q., Wang, X., Zhou, L., Wu, X., Liu, Y., Wu, G., Chen, K., He, S., Hu, W., Li, J-D., Li, J-X., Nian, G., Wang, L., Yang, J., Zhang, M., and Zhang, X.: CAS FGOALS-f3-L model datasets for CMIP6 historical Atmospheric Model Intercomparison Project simulation. Adv. Atmos. Sci., 36(8), 771-778, https://doi.org/10.1007/s00376-019-9027-8, 2019.

Kelley, M., G.A. Schmidt, L. Nazarenko, R.L. Miller, S.E. Bauer, R. Ruedy, G.L. Russell, I. Aleinov, M. Bauer, R. Bleck, V. Canuto, G. Cesana, Y. Cheng, T.L. Clune, B. Cook, C.A. Cruz, A.D. Del Genio, G.S. Elsaesser, G. Faluvegi, N.Y. Kiang, D. Kim, A.A. Lacis, A. Leboissetier, A.N. LeGrande, K.K. Lo, J.C. Marshall, S. McDermid, E.E. Matthews, K. Mezuman, L.T. Murray, V. Oinas, C. Orbe, C. Pérez García-Pando, J.P. Perlwitz, M.J. Puma, D. Rind, A. Romanou, D.T. Shindell, S. Sun, N. Tausnev, K. Tsigaridis, G. Tselioudis, E. Weng, J. Wu, and M. Yao, 2019: GISS-E2.1: Configurations and climatology. J. Adv. Model. Earth Syst., submitted.

Li, L., Yu, Y., Tang, Y., Lin, P., Xie, J., Song, M., Dong, L., Zhou, T., Liu, L., Wang, L., Pu, Y., Chen, X., Chen, L., Xie, Z., Liu, H., Zhang, L., Huang, X., Feng, T., Zheng, W., Xia, K., Liu, H., Liu, J., Wang, Y., Wang, L., Jia, B., Xie, F., Wang, B., Zhao, S., Yu, Z., Zhao, B., Wei, J.: The Flexible Global Ocean-Atmosphere-Land System Model Grid-Point Version 3 (FGOALS-g3): Description and Evaluation. Journal of Advances in Modeling Earth Systems, 12, e2019MS002012. https://doi.org/10.1029/2019MS002012, 2020.

Mauritsen, T., Bader, J., Becker, T., Behrens, J., Bittner, M., Brokopf, R., Brovkin, V., Claussen, M., Crueger, T., Esch, M., Fast, I., Fiedler,S., Fläschner, D., Gayler, V., Giorgetta, M., Goll, D. S., Haak, H., Hagemann, S., Hedemann, C., Hohenegger, C., Ilyina, T., Jahns,T., Jimenéz-de-la Cuesta, D., Jungclaus, J., Kleinen, T., Kloster, S., Kracher, D., Kinne, S., Kleberg, D., Lasslop, G., Kornblueh, L.,Marotzke, J., Matei, D., Meraner, K., Mikolajewicz, U., Modali, K., Möbis, B., Müller, W. A., Nabel, J. E. M. S., Nam, C. C. W.,Notz, D., Nyawira, S.-S., Paulsen, H., Peters, K., Pincus, R., Pohlmann, H., Pongratz, J., Popp, M., Raddatz, T. J., Rast, S., Redler, R.,Reick, C. H., Rohrschneider, T., Schemann, V., Schmidt, H., Schnur, R., 
Schulzweida, U., Six, K. D., Stein, L., Stemmler, I., Stevens,B., von Storch, J.-S., Tian, F., Voigt, A., Vrese, P., Wieners, K.-H., Wilkenskjeld, S., Winkler, A., and Roeckner, E.: Developments in theMPI-M Earth System Model version 1.2 (MPI-ESM1.2) and Its Response to Increasing CO2, J. Adv. Model. Earth Sy., 11, 998-1038, https://doi.org/10.1029/2018MS001400, 2019.

Ohgaito, R., Yamamoto, A., Hajima, T., O'ishi, R., Abe, M., Tatebe, H., Abe-Ouchi, A., and Kawamiya, M.: PMIP4 experiments using MIROC-ES2L Earth System Model, Geosci. Model Dev. Discuss., https://doi.org/10.5194/gmd-2020-64, in review, 2020.

Rackow, T., Goessling, H.F., Jung, T., Sidorenko, D., Semmler, T., Barbi, D., and Handorf, D.: Towards multi-resolution global climate modeling with ECHAM6-FESOM. Part II: climate variability. Climate Dynamics 50, 2369-2394, https://doi.org/10.1007/s00382-016-3192-6, 2018.

Seland, Ø., Bentsen, M., Seland Graff, L., Olivié, D., Toniazzo, T., Gjermundsen, A., Debernard, J. B., Gupta, A. K., He, Y., Kirkevåg, A., Schwinger, J., Tjiputra, J., Schancke Aas, K., Bethke, I., Fan, Y., Griesfeller, J., Grini, A., Guo, C., Ilicak, M., Hafsahl Karset, I. H., Landgren, O., Liakka, J., Onsum Moseid, K., Nummelin, A., Spensberger, C., Tang, H., Zhang, Z., Heinze, C., Iverson, T., and Schulz, M.: The Norwegian Earth System Model, NorESM2 - Evaluation of theCMIP6 DECK and historical simulations, Geosci. Model Dev. Discuss., https://doi.org/10.5194/gmd-2019-378, in review, 2020.

Shi, X., Yang, H., Danek, C., Lohmann, G.: AWI AWI-ESM1.1LR model output prepared for CMIP6 PMIP. Earth System Grid Federation, https://doi:10.22033/ESGF/CMIP6.9302, 2020a.

Shi, X., Yang, H., Danek, C., Lohmann, G.: AWI AWI-ESM1.1LR model output prepared for CMIP6 PMIP lgm. Earth System Grid Federation, https://doi:10.22033/ESGF/CMIP6.9330, 2020b.

Shi, X., Yang, H., Danek, C., Lohmann, G.: AWI AWI-ESM1.1LR model output prepared for CMIP6 PMIP lig127k. Earth System Grid Federation, https://doi:10.22033/ESGF/CMIP6.9331, 2020c.

Shi, X., Yang, H., Danek, C., Lohmann, G.: AWI AWI-ESM1.1LR model output prepared for CMIP6 PMIP midHolocene. Earth System Grid Federation, https://doi:10.22033/ESGF/CMIP6.9332, 2020d.

Sidorenko, D., Rackow, T., Jung, T., Semmler, T., Barbi, D., Danilov, S., Dethloff, K., Dorn, W., Fieg, K., Goessling, H.F., Handorf, D., Harig, S., Hiller, W., Juricke, S., Losch, M., Schroter, J., Sein, D.V., and Wang, Q.: Towards multi-resolution global climate modeling with ECHAM6-FESOM. Part I: model formulation and mean climate. Climate Dynamics 44, 757-780, https://doi.org/10.1007/s00382-014-2290-6, 2015.

Voldoire, A., Saint-Martin, D., Sénési, S., Decharme, B., Alias, A., Chevallier, M., Colin, J., Guérémy, J.-F., Michou, M., Moine, M.-P.,Nabat, P., Roehrig, R., Salas y Mélia, D., Séférian, R., Valcke, S., Beau, I., Belamari, S., Berthet, S., Cassou, C., Cattiaux, J., Deshayes, J.,Douville, H., Ethé, C., Franchistéguy, L., Geoffroy, O., Lévy, C., Madec, G., Meurdesoif, Y., Msadek, R., Ribes, A., Sanchez-Gomez, E.,Terray, L., and Waldman, R.: Evaluation of CMIP6 DECK Experiments With CNRM-CM6-1, J. Adv. Model. Earth Sy., 11, 2177-2213, https://doi.org/10.1029/2019MS001683, 2019.

Volodin, E. M., Mortikov, E. V., Kostrykin, S. V., Galin, V. Y., Lykossov, V. N., Gritsun, A. S., Diansky, N. A., Gusev, A. V., Iakovlev, N. G., Shestakova, A. A., and Emelina, S.V.: Simulation of the modern climate using the INM-CM48 climate model, Russian Journal of Numerical Analysis and Mathematical Modelling, 33, 367-374, https://doi.org/10.1515/rnam-2018-0032, 2018.

Williams, K., Copsey, D., Blockley, E. W., Bodas-Salcedo, A., Calvert, D., Comer, R., Davis, P., Graham, T., Hewitt, H. T., Hill, R., Hyder, P., Ineson, S., Johns, T. C., Keen, A. B., Lee, R. W., Megann, A., Milton, S. F., Rae, J. G. L., Roberts, M. J., Scaife, A. A., Schiemann, R., Storkey, D., Thorpe, L., Watterson, I. G., Walters, D. N., West, A., Wood, R. A., Woollings, T., and Xavier, P. K.: The Met Office Global Coupled model 3.0 and 3.1 (GC3.0 \& GC3.1) configurations, J. Adv. Model. Earth Sy., 10, 357-380, https://doi.org/10.1002/2017MS001115, 2018.

Yukimoto, S., Kawai, H., Koshiro, T., Oshima, N., Yoshida, K., Urakawa, S., Tsujino, H., Deushi, M., Tanaka, T., Hosaka, M., Yabu, S., Yoshimura, H., Shindo, E., Mizuta, R., Obata, A., Adachi, Y., and Ishii, M.: The Meteorological Research Institute Earth System ModelVersion 2.0, MRI-ESM2.0: Description and Basic Evaluation of the Physical Component, J. Meteorol. Soc. Jpn. Ser. II, 97, 931-965, https://doi.org/10.2151/jmsj.2019-051, 2019. 